\title{
Recent Progress of Membrane Distillation Technology Applied in Desalination
}

\author{
Xi Chen ${ }^{1, *}$ \\ ${ }^{1}$ Viterbi School of Engineering, University of Southern California,3551 Trousdale Pkwy, University Park, Los Angeles, CA 90007
}

\begin{abstract}
Membrane distillation (MD), as a biphasic separation method, has promising application prospect in seawater and brackish water desalination with desirable properties such as low energy consumption, cost-effective and high salt rejection, which has attracted more attention from researchers to explore its potential. In this paper, we summarize the operating mechanism and typical configurations of MD. Recent advances in three main research directions, including reforming membrane material, optimizing separation process and changing power source, are briefly overviewed. Additionally, challenges of future studies in MD are discussed at the end of this review, which purports to provide a reference for further development.
\end{abstract}

\section{Introduction}

As the consequence of over exploitation and increasing pollution of natural water, the source of potable use is depleted in recent years, especially part regions in midAmerica like Texas. Under continuous impact of global warming, around 1.8 billion population worldwide may suffer water shortage by 20251 . With the demand for fresh water still increasing in the past thirty years, reclamation from wastewater and desalination of seawater rose as popular application, which resulted in more than 15000 desalination plants installation until $2002[2,3]$.

Technologies used in desalination process can be classified into two types: thermal methods involving phase change processes and membrane method which related to single or multiple phases $[4,5]$. Common thermal techniques like multi-effect distillation (MED), multistage flash (MSF) and vapor compression (VC) provide higher treatment efficiency of salty water and larger average capacity of plant. However, requirement of massive amount of fuel in vaporizing increases the cost of thermal method. In addition, various membrane methods depended on hydrophobic material like pervaporation, membrane crystallization $(\mathrm{MCr})$ and membrane emulsification (ME) are developed in recent years [6]. Although most of them show promising properties when being applied in desalination, nearly half of the total capacity is still based on reverse osmosis (RO) technique, which needs high operation pressure and is energy intensive.

In order to resolve existing issues mentioned above, quantities of researches investigated different technologies and tried to develop a pragmatic and effective system to purify wastewater and seawater. Membrane Distillation (MD), as a thermally-driven treatment process, combines membrane-based separation and vapor pressure difference to achieve low energy consumption with low graded heat $[7,8]$. Since the hydrophobic membrane establishes a barrier that can discriminate the property of molecules (partial pressure in case of $\mathrm{MD}$ ), the operating temperature will generally be limited under $80^{\circ} \mathrm{C}$, which decreases power requirement compared with conventional distillation. In addition, MD process can improve the mechanical property and chemical resistance under relatively low costs and decline the extent of sensitivity to variable conditions like ph. With above advantages, MD becomes a promising technique to remove salt from high concentration solution and seawater [3].

In this work, we aim to provide a brief review of basic concept of MD process and summarize recent advances in relative technologies in terms of system configuration, membrane material and energy utilization. Moreover, current deficiencies of MD in desalination will be assessed at the end of this article in order to complete a comprehensive analysis of potential in future development.

\section{Principle and System Configuration of Membrane Distillation}

\subsection{Principle}

Membrane distillation utilizes semipermeable membrane to divide the system into two parts. One side is fed with water vapor that evaporates from heated flow under boiling point [2]. The other side can be designed as structure which allows the vapor to pass through the membrane to condense. The concentrates with pollutants $(\mathrm{NaCl}$ in desalination) will be retained by membrane

*Corresponding author: author@e-mail.org Correspondence: chen806@usc.edu 
while the water molecules can diffuse through it based on the partial pressure difference between both sides. The critical factor that prevents liquid phase penetrating membrane is the hydrophobicity of material. Depending on such characteristics, purified vapor can be easily separated from saline water.

\subsection{System Configuration}

The system of membrane distillation can be installed into four configurations, whose structure is shown in Fig.1. Based on relatively simple operation, the DCMD and AGMD will be more common in particle application [7].

\subsubsection{DCMD (Direct Contact Membrane Distillation)}

DCMD configuration allows liquid to flow on both side of membrane and the feed solution is heated on the surface of hot membrane. When it is converted into gas phase, the hydrophobic property of material and pressure difference will drive the vapor to transfer through the membrane and enter the cooler solution. Despite its widely application in desalination, there is still heat loss generated from conduction of membrane surface which cause a low energy utilization [2].

Research of [9] utilized polypropylene hollow fiber with three layers to improve the performance and stability operation under the temperature of $85^{\circ} \mathrm{C}$. [10] also developed a hollow-fiber membrane for DCMD to obtain a higher surface area and a larger flow rate.

\subsubsection{AGMD (Air Gap Membrane Distillation)}

Comparing to DCMD, a stagnant air should be inserted between hot membrane and cold plate to help evaporated water molecules condense on it. Although AGMD effectively reduces the energy loss through prevention of heat conduction, it also increases the resistance during transferring and produces a low yield of liquid [3].

\subsubsection{SGMD (Sweep Gas Membrane Distillation)}

If the system provides a sweep gas on the permeate side to carry vapor and transport it out to condense, then it can be called SGMD. [3] figured out that the dynamic air barrier in membrane module can decrease not only the heat loss but also the mass transfer resistance. When the volatile molecules are condensed outside the module, the efficiency of system is improved in a large scale. However, the defect of SGMD system mainly comes from the requirement of large condense container for quantities of sweep gas. [3] also mentioned a combined system (TSGMD) to obtain advantages of both configurations.

\subsubsection{VMD (Vacuum Membrane Distillation)}

VMD configuration will create a vacuum space on the permeate side of membrane to pump out the vapor to condense, which shares the similar structure with SGMD
[11]. Huge pressure difference between feeding side and vacuum can transfer the gas molecule more effectively.

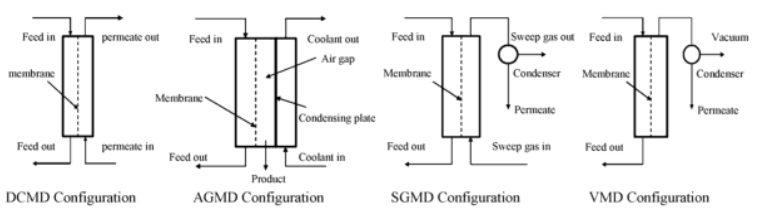

Fig.1. Different types of MD configurations [5].

\section{Recent Advance in Application of Membrane distillation}

Although membrane distillation currently has broad application in various fields like heavy metal removing of wastewater and valuable components recovery from juice, desalination, as its original design purpose, is still dominant in the utilization of MD. In addition, there were studies went steps further to research the desalination of seawater and brackish water [12].

\subsection{Membrane Material Studies of MD}

As the technical core of MD, membrane material has been studied in its numbers of properties including pore size, porosity and hydrophobicity. Higher flux and salt rejection can be obtained with optimized internal structure and roughness of membrane. This section sums up the most commonly used materials and their characteristics prepared for MD membranes in recent years, which achieve high hydrophobicity and bring better performance.

Various membranes were assessed in DCMD research of [13] based on the system setup in Fig.2. Materials of polyvinylidenefluoride (PVDF) and polytetrafluoroethylene (PTFE) were tested with/without support layer and pore size was also considered as a key factor during experiment. The results showed that, compared with microfiltration of PVDF, PTFE generated higher flux with high porosity [14], because the increase of porosity and pore size can amplify the mass transport coefficient of vapor in a reasonable magnitude. Their study also figured out that both permeability and energy consumption would be influenced by support layer and smaller thickness contributed to better performance. Due to a greater thermal efficiency, scrim backed membrane obtained better transfer properties than non-woven support ones.

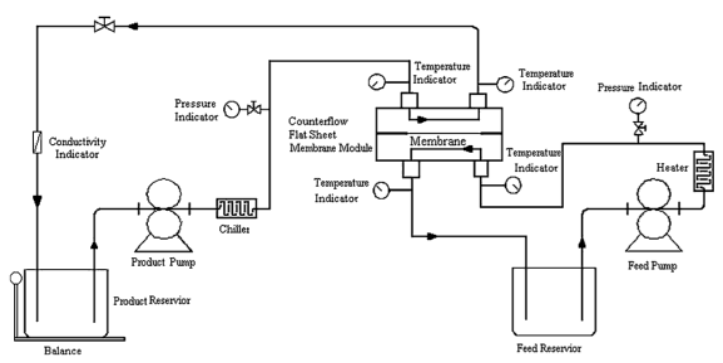

Fig.2. Schematic diagram of the experimental setup [13].

Flat sheet membrane with material of Poly (vinylidene fluoride-co-chlorotrifluoethylene) (PVDF- 
CTFE) was prepared with $\mathrm{LiCl}$ and non-solvent induced phase inversion (NIPS) by Zheng at el [6]. Since the research emphasized that hydrophobic membranes were required in MD desalination, characteristics of morphology and properties were assessed after NIPS. Results showed that shape of membrane surface would become irregular and porosity decreased with larger interconnected structure when the concentration of polymer was increased. Besides, highest roughness (SEM roughness index of 45.32) and the best MD performance (permeate flux of $20.65 \mathrm{~kg} /\left(\mathrm{m}^{2} \cdot \mathrm{h}\right)$ with a salt rejection higher than $99.95 \%$ ) could be reached when the concentration is $12 \%$. The hydrophobicity of membrane shared same tendency of roughness and relatively stable salt rejection was produced with high concentration invading membrane, which could be attributed to smaller pore size. Post treatment was performed with ethanol and sodium hydroxide $(\mathrm{NaOH})$ solution. Results showed that a second solution bath could be used for membrane modification to enhance the flux with the structure maintained.

Ren and co-works modified the polyvinylidene fluoride electrospum nanofiber membrane (PVDF ENM) with fluorosilanized membrane which is coated by $\mathrm{TiO} 2$ to obtain super-hydrophobicity used in DCMD of membrane distillation process [1]. Optimized properties including roughness $(4.63 \mu \mathrm{m})$, thickness $(55 \mu \mathrm{m})$, pore size (average $0.81 \mu \mathrm{m})$ and porosity $(57 \%)$ were achieved on membrane surface, which increased the attraction of PVDF ENM as potential material for DCMD. Wetting resistance was increased with the increase of roughness and water drops could be resisted effectively by such structure, which is shown in Fig.3. Finally, high performance at mean flux of 73.4 LMH and salt ( $\mathrm{NaCl}$ solution) rejection of $99.99 \%$ were observed in experiment, which demonstrated that PVDF ENM modified by TiO2-FTCS could be considered as a candidate of MD desalination.

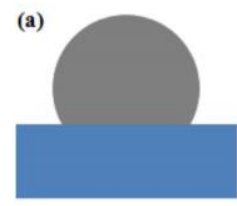

flat membrane Commercial PVDF membrane

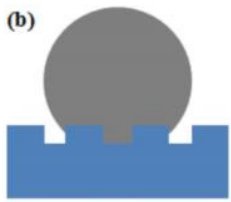

rougher membrane Unmodified PVDF ENM

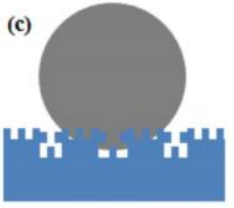

roughest membrane $\mathrm{TiO}_{2}$-FTCS modified PVDF ENM
Fig.3. simulated diagram of contact angles for membranes [1].

PTFE particles were incorporated in dope solution to form a macrovoids-free and high hydrophobic layer attached on PVDF/PTFE composite hollow fiber for seawater desalination via DCMD [15]. The thickness of outer-layer with macrovoid-free morphology was around $13 \mu \mathrm{m}$ under $30 \mathrm{wt} \%$ concentration of PTFE in dope solution. Although the macrovoids structure was not desired in membrane application, dope solution with PTFE could help to suppress it and decline the diffusion. Same material of inner and outer layer increased the compatibility between them and formed an unstratified membrane, which is shown in Fig.4. With the relatively thinner outer layer, mass transfer resistance of liquid was declined in a large scale. Compared with single-layer
PVDF/PTFE hollow fiber, dual-layer could increase the flux at $24 \%$. Finally, 100 hours' stable operation of desalination with dual-layer PVDF/PTFE could be found in the result of experiment and almost $100 \%$ of salt rejection was achieved.

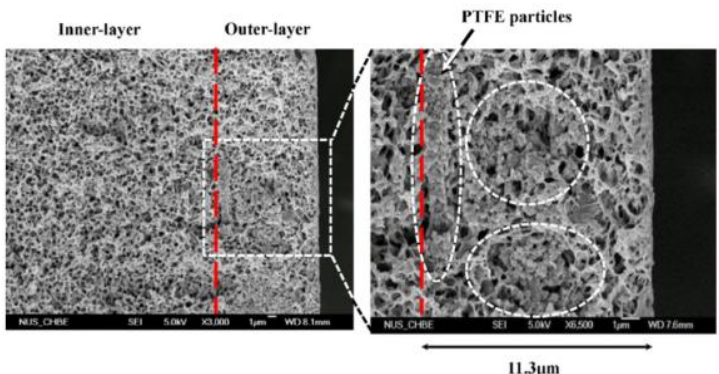

Fig.4. distribution of PTFE particles in the outer-layer polymer matrix [15].

In order to reduce the toxicity of membrane for seawater desalination in manufacture, researchers considered to utilize triethyl phosphate (TEP) as raw material to produce PVDF hollow fiber [16]. Compared with conventional process, the combination of PVDF/TEP could obtain more sponge-like structure with higher porosity, which, on average, could be higher than $80 \%$. In addition, membrane produced in this study gained higher viscosity and decreased hydrophilia of water to reach effective performance at 12 to 88 weight percent of PVDF/TEP. Finally, flux of $20 \mathrm{~kg} /\left(\mathrm{m}^{2} \cdot \mathrm{h}\right)$ and salt rejection of $99.99 \%$ made TEP a potential, non-toxic solvent to produce membrane applied in DCMD of seawater desalination.

\subsection{Configuration studies for MD}

According to the difference of fluid transportation, the configurations of MD are mainly categorized into four types, which are mentioned in previous introduction. Recently, more new configurations with sophisticated combining form have been proposed to improve the separation performance and reduce the operation cost. Diverse contact and interactive mode increase the efficiency of applying energy into pumping fluid and reduce thermal loss or resistance of mass transferring. This section lists part of representative installation of MD systems newly developed with higher flux of permeate.

Safavi and co-workers investigated the possibility of desalination of high concentration $\mathrm{NaCl}$ Solution with VMD. The new module was optimized in their research to achieve greater flux and consistent performance of flat sheet membrane with material of polypropylene and pore size of $0.2 \mu \mathrm{m}$. Configuration of membrane model and VMD system can be shown in Fig.5 and Fig.6. The results indicated that temperature and flow rate had positive effect on permeate flux while the increase of concentration or vacuum pressure would lead to the decrease of flux. In addition, the research also tested the contribution percent of different factors and found out that vacuum pressure could impact water quality in the largest scale. Under optimum operating, VMD performance at flux of $14.4 \mathrm{~kg} /\left(\mathrm{m}^{2} \cdot \mathrm{h}\right)$ and average 
electrical conductivity (EC) of $2.49 \mu \mathrm{S} / \mathrm{cm}$ can be obtained.

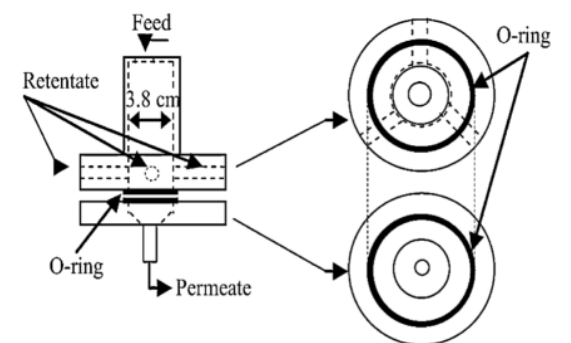

Fig.5. Membrane module [17].

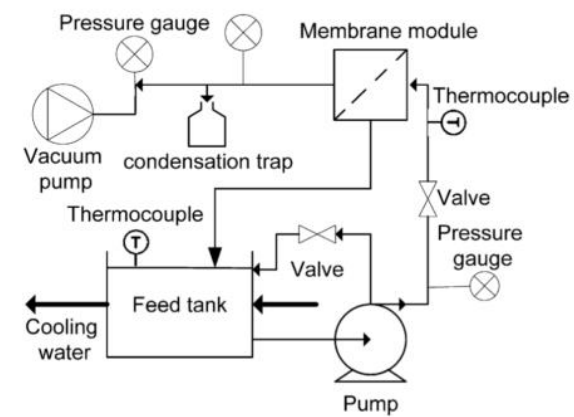

Fig.6. Schematic diagram of VMD setup [17].

Different salt concentration of city water was heated and employed as hot brine to be desalinated with DCMD [18]. Various configuration of hollow fiber was the research emphasis and horizontal crossflow modules with around 0.61 or $0.66 \mathrm{~m}^{2}$ surface area were adopted. Different from traditional flat sheet DCMD, the research developed crossflow installation in each single column with ten rows and the interaction of liquid on both sides of membrane can be shown in Fig.7 and Fig.8. They also built mathematical model in multiple modules distillation to test flux and performance, whose results indicated that higher temperature, interstitial velocity and concentration of $\mathrm{NaCl}$ helped to increase the vapor flux and most curves could fit the experimental data. Average flux of water vapor ranged from 15 to $33 \mathrm{~kg} /$ $\left(\mathrm{m}^{2} \cdot \mathrm{h}\right)$ in multiple modules could be obtained with 6-10 modules employed. Highest flux with $55 \mathrm{~kg} /\left(\mathrm{m}^{2} \cdot \mathrm{h}\right)$ was achieved with two modules in series. The concentration of salt in effluent could be limited under $1 \mathrm{ppm}$ with the original solution at $10 \% \mathrm{NaCl}$. In addition, no membrane contamination was occurred of the membrane modules.

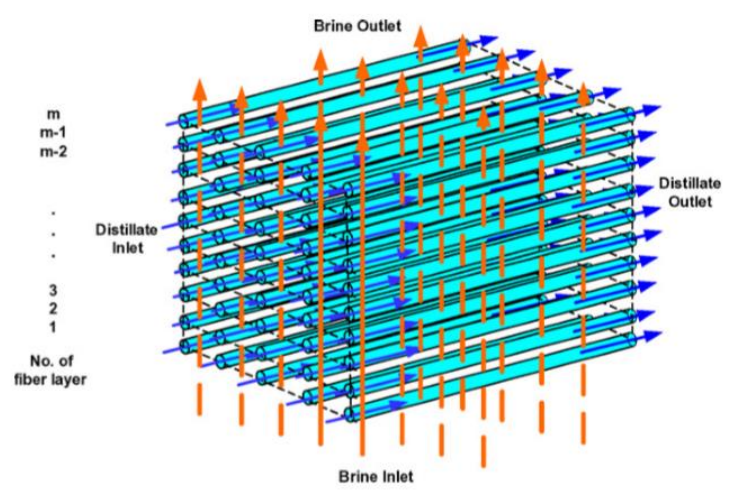

Fig.7. Arrangement of fibers in a DCMD module [18].

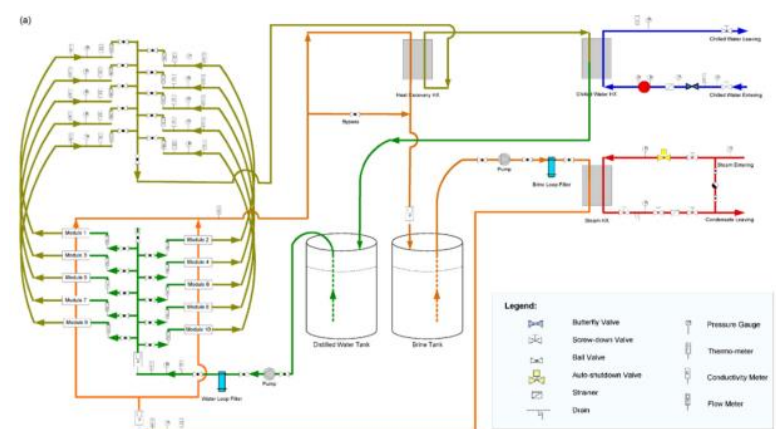

Fig.8. Schematic of DCMD pilot plant [18].

Energy efficiency of common single-stage MD configurations, including the direct contact, air gap and vacuum, was tested based on desalination process [19]. Gained output ratio (GOR), as the ratio between heat evaporated on unit mass of water and energy consumption, can indicate the performance of system. Different model equations which determine rate limiting process had effect on GOR and the most significant limitation was observed in VMD system because of its inherent property of low pressure. Temperature cannot reach a high value in vacuum condenser and, as a result, difficulty appeared in heating brine and recovering heat effectively. In contrast, DCMD and AGMD showed potential ability to achieve high GOR under corresponding operation condition. Decreasing the flux, resistance of mass transfer and width in AGMD can also help to increase the GOR.

Cath and co-workers developed and evaluated a new MD configuration and membrane module in improving desalination performance of $\mathrm{NaCl}$ and synthetic sea water [20]. Three configurations of membrane cell with four different materials were tested in research. The conventional counter currently cell collected the baseline data while other two configurations combined DCMD and vacuum pump were used to reduce the energy inefficiency. New design of installation is shown in the Fig.9. Better mix pattern-provided system with less temperature polarization when higher permeability increased mass transport. More than $99.9 \%$ of salt rejection can be obtained in all experiment. Flow velocity changed from 0.7 to $2.1 \mathrm{~m} / \mathrm{s}$ when switching the positive pressure $(108 \mathrm{kPa})$ to negative one $(94 \mathrm{kPa})$ and finally contributed to $15 \%$ increase of flux in DCMD/VMD system. Similar results could be obtained in deepening level of vacuum and the water flux can increase up to $84 \%$ in the deepest vacuum situation.

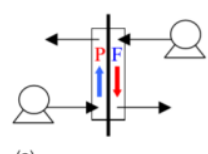

(a)

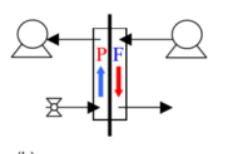

(b)

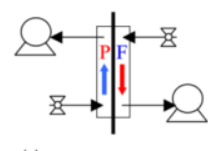

(c)
Fig.9. DCMD configuration: (a) traditional, (b) vacuum on the permeate side, (c) vacuum on both sides [20].

Inside-out and outside-in configurations with hollow fiber module were assessed and compared in VMD process of desalination of seawater [21]. Pure water, $\mathrm{NaCl}$ solutions with concentration ranged from $15 \mathrm{~g} / \mathrm{L}$ to $300 \mathrm{~g} / \mathrm{L}$ and two fiber with different materials and structure were prepared for this experiment. $0.08 \mathrm{~m}^{2}$ surface area of PVDF fiber was installed in module 1 while $0.2 \mathrm{~m}^{2}$ area of polyethylene fiber was used in 
module 2 and the configurations of each module is shown in Fig.10. Results revealed that temperature had positive contribution to the permeability while the increase of salt concentration would decrease the flux. The flux lost $30 \%$ of flux losing was shown when the concentration of $\mathrm{NaCl}$ was added up to $300 \mathrm{~g} / \mathrm{L}$. In addition, module 1 showed similar permeability with two configurations (inside-out and outside-in). However, slight difference in permeability with two configurations could be found in module 2 . Inside-out system obtained $2.2 \times 10-7$ s.mol1/2.m-1.kg- $1 / 2$ of pure water and the data of outside-in configuration could only reach $1.3 \times 10-7$ s.mol1/2.m-1.kg-1/2, which might be caused by the difference of location of interface between liquid and vapor in the membrane pores.

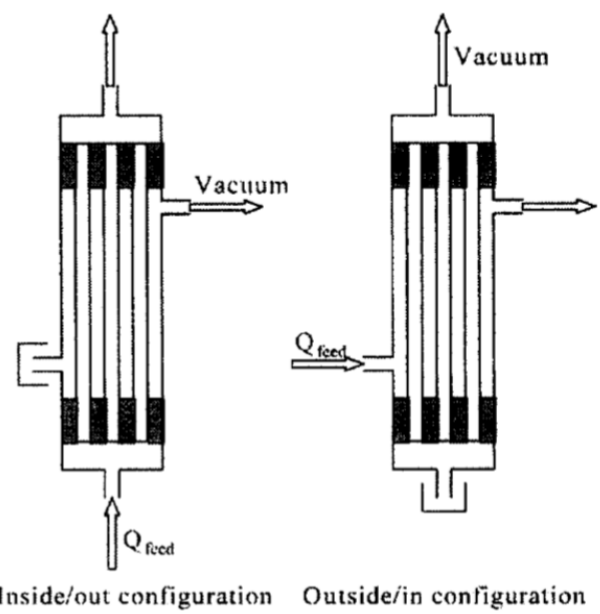

Fig.10. Module of membrane distillation [21].

\subsection{Energy consumption studies for MD}

Since the heating requirement of MD is relatively low compared with conventional distillation and system can be operated under moderate temperature range (under $85^{\circ} \mathrm{C}$ ), energy source supply like solar power and lowgrade waste can satisfy the need with suitable scale. This section summarizes part of applications of solar panel equipped in MD system and process combined with waste heat under intermittency operation, which will be significant in remote area without power network [22, 23].

There is a research studying the seawater desalination by innovative solar-powered membrane distillation system (MEDESOL) project, which utilized innovative solar-powered MD to complete desalination of seawater [24]. The research tried to evaluate the efficiency and cost of desalination with solar multi-stage MD, whose concept involves integrating five steps membrane treatment in capacity range from 0.5 to $50 \mathrm{~m}^{3}$. Their work provided possibility of desalination in region without electric grid and helped to decrease the cost under a relatively high efficiency. The most important achievement was that both batch scale and industry application were tested based on comprehensive project which combined advanced laboratory and experimental condition.
Desalination systems compacted with membrane distillation and driven by solar thermal force were developed in regions with scarce potable water [25]. The capacity of small system with low fresh water ranged from 100 to $500 \mathrm{~L} /$ day and compact configuration was designed, which is shown Fig.11. In addition, the twoloop system will be suitable for capacity of $10 \mathrm{~m}^{3} / \mathrm{day}$ in larger system and the installation can be seen in Fig.12. Results showed that, in compact system, temperature at evaporator inlet started from $55{ }^{\circ} \mathrm{C}$ and reached the peak of $85{ }^{\circ} \mathrm{C}$. Maximum flow of distillation was $15 \mathrm{~L} / \mathrm{h}$ and the volume which could be collected in a single day was $75 \mathrm{~L}$. As for two-loop system, the inlet temperature of heat exchanger is controlled at $78{ }^{\circ} \mathrm{C}$ while the outlet kept rising. Under such conditions, 75 to $80 \mathrm{~kg} / \mathrm{h}$ of distillation flow could be gained and $1200 \mathrm{~kg}$ of accumulated liquid was collected after six and a half hours' operation.

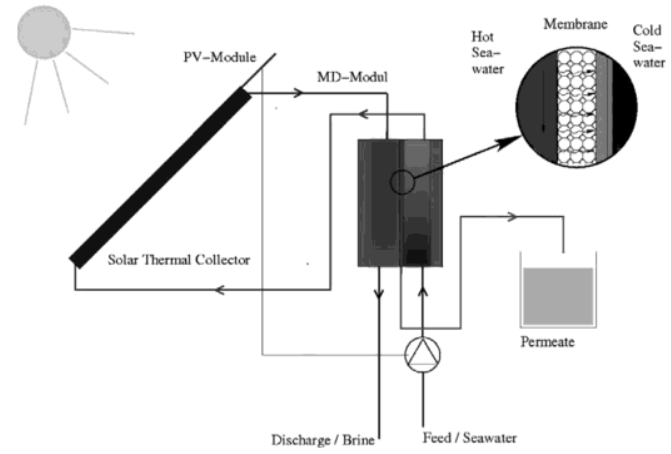

Fig.11. Principle and installation of compact MD system [25].

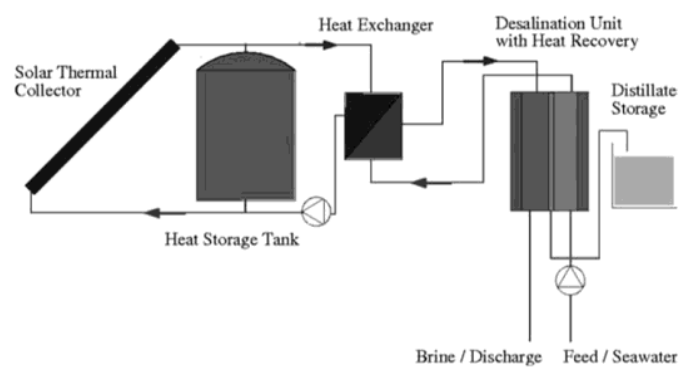

Fig.12. Principle and installation of two-loop MD system [25].

In order to establish medium scale and potent desalination for water supplying, three distinctive plants with one waste heat and two solar thermal driven systems were compared [26]. First, Pantelleria system was designed to collect heat from a diesel engine and transfer it into MD-modules. Pantelleria can be operated all the time under 65-75 centigrade without need of solar power. Low salinity was obtained by lower driven force based on temperature difference in long channel. However, this system provided less practical experience for application. Namibia system in Amarika selected to adsorb radiation from PV plate and utilized such power to treat the brackish water with beneficial energy consumption, which, however, can only be used under low salinity condition. In contrast, Gran Canary plant purified the concentration of $35 \mathrm{~g} / \mathrm{kg}$ seawater as a partner of MEDIRAS project. Both Namibia and Gran Canary required a storage under fluctuating solar supply and the operating interval for the former was $10 \mathrm{~s}$ while the latter was $15 \mathrm{~s}$. Without extra post treatment, effluent 
of Namibia and Gran Canary system was discharged for potable usage and irrigation, respectively.

Two distinct MD desalination modules were built and tested in practical situation by Keppel Seghers in Singaporean [27]. Both were based on AGMD system coupled with static solar collector and achieved 2400 hours' operation with $9 \mathrm{~m}^{2}$ membrane surface, respectively, in two years' range. Single (module A) and in series (module B) configurations were mainly compared in research. Results showed that module B always had $2 \%$ higher recovery ratio compared with module A and maximum difference could reach $4.54 \%$, which indicated that better performance could be obtained with multi-stage system in up-scaling. Higher temperature $\left(>50{ }^{\circ} \mathrm{C}\right)$, suitable feed flow rate $(26 \mathrm{~L} / \mathrm{min})$ and lower initial concentration $(1 \mathrm{~g} / \mathrm{L})$ can help to increase the performance. In addition, maximum flux of $3.5 \mathrm{~L} /\left(\mathrm{m}^{2} \cdot \mathrm{h}\right)$ was achieved and average salt rejection was above $99.8 \%$.

Direct and indirect system arrangement of intermittent and variable temperature waste heat are evaluated in current study of MD operation [28]. The main difference between the direct and indirect configurations is that single loop is used in the former while two separate loops in the latter, which can be shown in Fig.13. The result showed that, although there was only $6.7 \%$ higher temperature of direct arrangement, large difference of $17.3 \%$ in average water flux between two configurations would occur, depending on the exponential relationship acting on vapor pressure. However, indirect arrangement could reserve more heat at steady state and maintain a relatively flat change during interruption of heat source. In addition, with a smaller drop of water flux after long-term operation, indirect arrangement could generate more water when using waste heat at high intermittency (90\%). Results showed that low intermittency $(12.5 \%)$ was suitable for direct arrangement and high intermittency $(87.5 \%)$ for indirect one when the operating heat source was intermittent. When the operating heat source is variabletemperature waste heat, even the direct arrangement has greater water flux, the indirect one has forecasting feature which makes contribution in system control and water availability prediction.

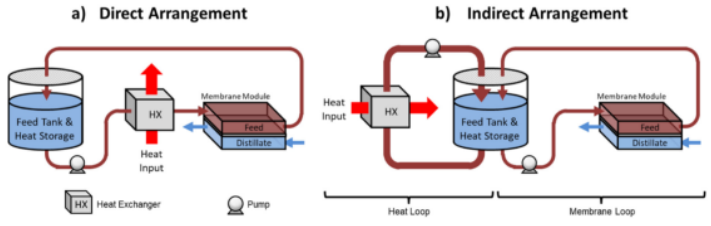

Fig.13. direct and indirect system arrangement [28].

\section{Conclusion}

MD based on second generation application of membrane technology can utilize the pressure difference of vapor to achieve salt removing from solution under low energy consumption. High desalination rate, simple configuration and alternative heating source make MD become an attractive process for purifying seawater and wastewater. Current researches focus on the improvement of material preparation, system construction and energy exploiting. In order to establish an effective treatment method, properties related to flux increase, salt rejection maximization and relatively large-scale application under available energy supply should be carefully taken into consideration.

As for membrane materials, polyvinylidenefluoride (PVDF), which has high hydrophobicity, can be used as a basic carrier to synthesize multiple composite material to intensify the selectivity of MD. Polymer like PTFE and CTFE can be combined with it to refine surface structure of membrane. In addition, extreme water repellency showed on nanofibers coated with $\mathrm{TiO} 2$ and nontoxicity can be obtained from TEP as raw material. For the installation, direct contact and vacuum membrane distillation are most commonly studied and usually combined with various systems to enhance the effectiveness. Generally, the increase of flow velocity and temperature can expand flux among different configurations. Deeper vacuum situation and multiple stage system can also contribute to greater permeability of MD. In terms of energy usage, consuming through solar and waste heat is evaluated in experimental scale and the test of intermittent operation also indicates the practicability. Most researches show that solar power is enough to support small and medium-sized MD system during sufficient sunshine period. Variable waste heating can even satisfy the requirement of temperature range and maintain a relatively stable long-term operation.

Based on above discussion, though great progress regarding to MD process has been made in recent years, commercial scale MD plant without regional restriction still needs to be developed by technique modification. Disadvantages such as membrane fouling, which requires periodic treatment or replacement, still need to be improved. Moreover, the utilization efficiency of energy restrains the possibility of large size application in industrialized desalination. Membrane material can be further analyzed on mechanism to avoid fouling and reduce cost. More testing of configuration with stereo structure should be done for obtaining higher mass transfer capacity. Additionally, economical and effective methods of desalination coupled with multiple energy source have to be developed for those energy lacking areas.

\section{Reference}

1. Ren, L.-F., et al., TiO2-FTCS modified superhydrophobic PVDF electrospun nanofibrous membrane for desalination by direct contact membrane distillation. Desalination, 2017. 423: p. 111.

2. Wang, L.K., et al., Membrane and desalination technologies. Vol. 13. 2008: Springer.

3. Alkhudhiri, A., N. Darwish, and N. Hilal, Membrane distillation: a comprehensive review. Desalination, 2012. 287: p. 2-18.

4. Karagiannis, I.C. and P.G. Soldatos, Water desalination cost literature: review and assessment. Desalination, 2008. 223(1-3): p. 448-456. 
5. El-Bourawi, M., et al., A framework for better understanding membrane distillation separation process. Journal of membrane science, 2006. 285(12): p. 4-29.

6. Zheng, L., et al., Preparation, evaluation and modification of PVDF-CTFE hydrophobic membrane for MD desalination application. Desalination, 2017. 402: p. 162-172.

7. Lawson, K.W. and D.R. Lloyd, Membrane distillation. Journal of membrane Science, 1997. 124(1): p. 1-25.

8. Al-Obaidani, S., et al., Potential of membrane distillation in seawater desalination: thermal efficiency, sensitivity study and cost estimation. Journal of Membrane Science, 2008. 323(1): p. 8598.

9. Li, B. and K.K. Sirkar, Novel membrane and device for direct contact membrane distillation-based desalination process. Industrial \& Engineering Chemistry Research, 2004. 43(17): p. 5300-5309.

10. ingh, D., et al., Novel cylindrical cross-flow hollow fiber membrane module for direct contact membrane distillation-based desalination. Journal of Membrane Science, 2018. 545: p. 312-322.

11. Li, B. and K.K. Sirkar, Novel membrane and device for vacuum membrane distillation-based desalination process. Journal of Membrane Science, 2005. 257(1-2): p. 60-75.

12. Wang, P. and T.-S. Chung, Recent advances in membrane distillation processes: Membrane development, configuration design and application exploring. Journal of membrane science, 2015. 474: p. 39-56.

13. Zhang, J., et al., Identification of material and physical features of membrane distillation membranes for high performance desalination. Journal of Membrane Science, 2010. 349(1-2): p. 295-303.

14. Adnan, S., et al., Commercial PTFE membranes for membrane distillation application: effect of microstructure and support material. Desalination, 2012. 284: p. 297-308.

15. Teoh, M.M., T.-S. Chung, and Y.S. Yeo, Dual-layer PVDF/PTFE composite hollow fibers with a thin macrovoid-free selective layer for water production via membrane distillation. Chemical engineering journal, 2011. 171(2): p. 684-691.

16. Chang, J., et al., Using green solvent, triethyl phosphate (TEP), to fabricate highly porous PVDF hollow fiber membranes for membrane distillation. Journal of Membrane Science, 2017. 539: p. 295304.

17. Safavi, M. and T. Mohammadi, High-salinity water desalination using VMD. Chemical Engineering Journal, 2009. 149(1-3): p. 191-195.

18. Song, L., et al., Pilot plant studies of novel membranes and devices for direct contact membrane distillation-based desalination. Journal of Membrane Science, 2008. 323(2): p. 257-270.

19. Summers, E.K. and H.A. Arafat, Energy efficiency comparison of single-stage membrane distillation (MD) desalination cycles in different configurations. Desalination, 2012. 290: p. 54-66.

20. Cath, T.Y., V.D. Adams, and A.E. Childress, Experimental study of desalination using direct contact membrane distillation: a new approach to flux enhancement. Journal of Membrane Science, 2004. 228(1): p. 5-16.

21. Wirth, D. and C. Cabassud, Water desalination using membrane distillation: comparison between inside/out and outside/in permeation. Desalination, 2002. 147(1-3): p. 139-145.

22. Lu, H., J.C. Walton, and A.H. Swift, Desalination coupled with salinity-gradient solar ponds. Desalination, 2001. 136(1-3): p. 13-23.

23. Saffarini, R.B., E.K. Summers, and H.A. Arafat, Technical evaluation of stand-alone solar powered membrane distillation systems. Desalination, 2012. 286: p. 332-341.

24. Gálvez, J.B., L. García-Rodríguez, and I. MartínMateos, Seawater desalination by an innovative solar-powered membrane distillation system: the MEDESOL project. Desalination, 2009. 246(1-3): p. 567-576.

25. Koschikowski, J., et al., Experimental investigations on solar driven stand-alone membrane distillation systems for remote areas. Desalination, 2009. 248(13): p. 125-131.

26. Schwantes, R., et al., Membrane distillation: Solar and waste heat driven demonstration plants for desalination. Desalination, 2013. 323: p. 93-106.

27. Guillén-Burrieza, E., et al., Experimental evaluation of two pilot-scale membrane distillation modules used for solar desalination. Journal of Membrane Science, 2012. 409: p. 264-275.

28. Gustafson, R.D., S.R. Hiibel, and A.E. Childress, Membrane distillation driven by intermittent and variable-temperature waste heat: System arrangements for water production and heat storage. Desalination, 2018. 448: p. 49-59. 